\title{
Article
}

\section{Novel state-space realization generalized from turbine blade modeling}

\author{
Andrew van Paridon ${ }^{1}$, Timothy Sands $2, *$, (D) \\ 1 Sibley School of Mechanical and Aerospace Engineering, Cornell University, New York 14853, USA \\ 2 Department of Mechanical and Aerospace Engineering, Naval Postgraduate School, Monterey 93943, USA \\ * Correspondence: tasands@nps.edu
}

1 Abstract: Mathematical models across the applied sciences often utilize a standard methodological representation called a state variable formulation more commonly referred to as state space form.

Recent research in unmanned underwater vehicle motor turbine blade thermal modeling for

fatigue-life is generalized here permitting the proposed novel state space from to be applied to

electrodynamics, motion mechanics, and many other disciplines. Proposed here is a very compact

form inherently representing time variance, with a convenient presentation of dynamic variables applicable to all proper transfer functions, where all the distinct, real poles, zeros and gain of the transfer function appear as explicit components in the state space. The resulting manifestation simplifies utilization of the state space methods broadly across the applied sciences.

Keywords: transfer function; state-space; realization; conversion

Finite systems of deterministic ordinary nonlinear differential equations may be designed to represent forced dissipative hydrodynamic flow. Solutions of these equations can be identified with trajectories in phase space.

\section{— Edward Lorenz, Abstract from his landmark paper [1]}

\section{Problem Statement}

Unmanned underwater vehicle turbine powered charging system and methods were recently presented in [2], while reference [3] introduced a state-space realization used to model temperature modes of turbine discs in order to correlate fatigue-life degradation. Figure 1 from that prequel is included as figure 1 in this manuscript. The full mathematical solution to the general case was found subsequently, and is presented here in full for the first time in a very compact form to represent dynamic systems that neatly separates past from future, [4]. Such representation is very often obtained through linearization of nonlinear differential equations around a logical initial operating point, [5], and is ubiquitously applicable to power systems [5], robotic manipulators [6] and balance control [7], spacecraft [8], small signals [9], process tomography of fluid flows [10], and are foundation to general problems of stability and control [11] so as to become key to the so-called control diagram method. Thus, the method proposed here has generic applicability beyond unmanned underwater vehicle turbine blades. This research introduces a method to produce state space forms with blatant display of all distinct real poles, zeros, and gain applicable to all proper transfer function ratios of system outputs to inputs.

Consider the single-input-single-output transfer function in pole-zero form;

$$
G(s)=\frac{\prod_{i=1}^{M}\left(s-z_{i}\right)}{\prod_{j=1}^{N}\left(s-p_{i}\right)} \cdot k U(s)
$$


It is useful to be able to construct a state-space realization such that the numerical values of the poles, zeros and gain appear explicitly in the matrices of;

$$
\begin{aligned}
\dot{x}(t) & =\mathbf{A} x(t)+\mathbf{B} u(t) \\
y(t) & =\mathbf{C} \boldsymbol{x}(t)+\mathbf{D} u(t)
\end{aligned}
$$

A general form of the realization is sought for all proper transfer functions, where the number of poles $N$ is equal to or great than then number of zeros $M$.
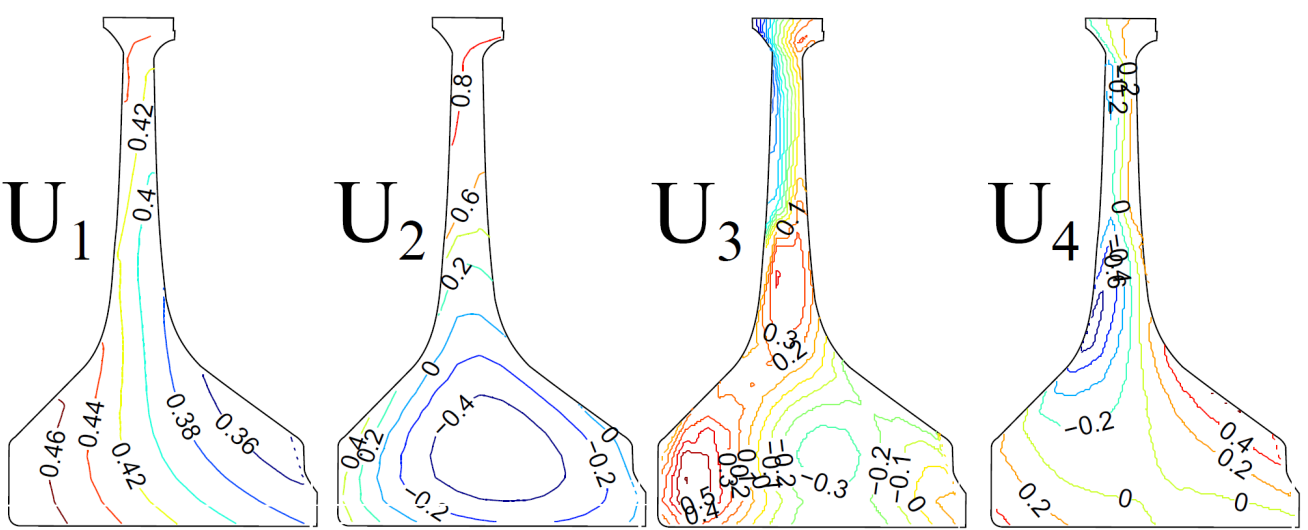

Figure 1. Thermal modes of a turbine disc. Each mode was associated with an element from $\mathbf{C}$ in order build a complete temperature simulation.

With this introduction to the problem to be solved, the manuscript proceeds first with a brief introduction to the scope of the solution (categorized into four functions of interest) and necessary introduction to terminology and nomenclature of the effort. Next, each special case in the scope of the solution is discussed sequentially, developing the proposed methodology along the way. Lastly, comparisons are made with canonical forms ubiquitously in use. Examples conversions are included in an appendix.

\section{Overview of Solution}

We see that each surface is really a pair of surfaces, so that, where they appear to merge, there are really four surfaces. Continuing this process for another circuit, we see that there are really eight surfaces etc and we finally conclude that there is an infinite complex of surfaces, each extremely close to one or the other of two merging surfaces.

\section{— Edward Lorenz}

An intermediate thought about some surface in state space, while evolving his prototype model of chaos. [12]

\subsection{The 4 Main Categories}

The Pole-Zero-Difference form is named for the off-diagonal terms in the A matrix. Realizations in this form have been found for all proper transfer functions and will be presented here. However, the algebra becomes incredibly expansive to prove in the general case. As such, this paper will explore the solution across a number of cases in order to build the methodology of the proof. The Appendix provides a list of simple cases for quick reference. Broadly, there are 4 categories of functions of interest, where;

1. there are no zeros, $M=0$.

2. the number of zeros is anything up to $M<N-1$.

3. the number of zeros is exactly $M=N-1$.

4. the number of poles and zeros are equal, $M=N$.

The full solution for the last two categories is shown in summary against other canonical forms in Section 4. There is significant overlap between these categories for B, C, D matrices, with most of the differences confined to the A matrix. 


\section{2.2. The construction of $\mathbf{B}, \mathbf{C}, \mathbf{D}$}

The matrix $\mathbf{B}$ is constructed the same for all categories; as a column vector of length $N$ such that;

$$
\mathbf{B}=\left[\begin{array}{lllll}
k & 0 & 0 & \ldots & 0
\end{array}\right]^{\top}
$$

In the singular case where $N=1$;

$$
\mathbf{B}=k
$$

63 Similarly, matrix $\mathbf{C}$ is a row vector of length $N$. For categories 1 and 2;

$$
\mathbf{C}=\left[\begin{array}{lllll}
0 & \ldots & 0 & 0 & 1
\end{array}\right]
$$

for category 3 , where $M=N-1$;

$$
\mathbf{C}=\left[\begin{array}{lllll}
1 & \ldots & 1 & 1 & 1
\end{array}\right]
$$

and for category 4 , where $M=N$, matrix $\mathbf{C}$ is more complicated;

$$
\mathbf{C}=\left[\begin{array}{llll}
\sum_{i=1}^{N}\left(p_{i}-z_{i}\right) & \ldots & \sum_{i=N-1}^{N}\left(p_{i}-z_{i}\right) & p_{N}-z_{N}
\end{array}\right]
$$

64 This case is covered more fully below.

65

\section{2.3. The construction of $\mathbf{A}$}

The matrix $\mathbf{A}$ is always lower triangular, and constructed by placing the poles ( $p_{1}$ to $p_{N}$ ) on the main diagonal of a square matrix. For the first category, the case of no zeros, 1's are placed on the subdiagonal;

$$
\mathbf{A}=\left[\begin{array}{cccccc}
p_{1} & 0 & 0 & 0 & \ldots & 0 \\
1 & p_{2} & 0 & 0 & & \\
0 & 1 & p_{3} & 0 & & \\
0 & 0 & 1 & p_{4} & & \\
\vdots & & & \ddots & \ddots & 0 \\
0 & & \ldots & 0 & 1 & p_{N}
\end{array}\right]
$$

Where the full state-space would be;

$$
\left[\begin{array}{cc}
\mathbf{A} & \mathbf{B} \\
\mathbf{C} & \mathbf{D}
\end{array}\right]=\left[\begin{array}{ccccccc}
p_{1} & 0 & 0 & 0 & \ldots & 0 & k \\
1 & p_{2} & 0 & 0 & & & 0 \\
0 & 1 & p_{3} & 0 & & & 0 \\
0 & 0 & 1 & p_{4} & & & 0 \\
\vdots & & & \ddots & \ddots & 0 & 0 \\
0 & & \ldots & 0 & 1 & p_{N} & 0 \\
0 & & \ldots & 0 & 0 & 1 & 0
\end{array}\right]
$$

This would represent the transfer function;

$$
G(s)=\frac{1}{\prod_{j=1}^{N}\left(s-p_{i}\right)} \cdot k U(s)
$$

${ }_{68}$ This particular form is already well known, and represents the complete set of functions

69 from category 1 . It is trivial to prove so not considered further.

For category 2, where the number of poles and zeros is strictly $N>M>0$, we first consider simply $M=1$, where the single zero is defined as $z_{1}$. This is placed in A on the 
second row under the first pole, as the pole-zero difference $\left(p_{2}-z_{1}\right)$. Additionally, on the row below this (the third row, i.e. row number $M+2$ ) place 1's up until the main diagonal;

$$
\mathbf{A}=\left[\begin{array}{cccccc}
p_{1} & 0 & 0 & 0 & \ldots & 0 \\
p_{2}-z_{1} & p_{2} & 0 & 0 & & \\
1 & 1 & p_{3} & 0 & & \\
0 & 0 & 1 & p_{4} & & \\
\vdots & & & \ddots & \ddots & 0 \\
0 & & \ldots & 0 & 1 & p_{N}
\end{array}\right]
$$

For a second zero, $z_{2}$, the pattern continues, but now there are two columns with the pole-zero difference $\left(p_{3}-z_{2}\right)$. Again, the row $M+2$ will have 1 's up until the main diagonal;

$$
\mathbf{A}=\left[\begin{array}{cccccc}
p_{1} & 0 & 0 & 0 & \ldots & 0 \\
p_{2}-z_{1} & p_{2} & 0 & 0 & & \\
p_{3}-z_{2} & p_{3}-z_{2} & p_{3} & 0 & & \\
1 & 1 & 1 & p_{4} & & \\
\vdots & & & \ddots & \ddots & 0 \\
0 & & \ldots & 0 & 1 & p_{N}
\end{array}\right]
$$

So, for each subsequent zero up to $z_{M}$, the rows are constructed as;

$$
\mathbf{A}=\left[\begin{array}{cccccccc}
p_{1} & & & & & & & \\
p_{2}-z_{1} & p_{2} & & & & & & \\
\vdots & & \ddots & & & & & \\
& & {[-} & \mathbf{A}_{M+1} & -] & & & \\
1 & \ldots & 1 & 1 & p_{M+2} & & & \\
0 & & \cdots & 0 & 1 & p_{M+3} & & \\
\vdots & & & & & & \ddots & \\
0 & & & \ldots & & 0 & 1 & p_{N}
\end{array}\right]
$$

where the $M+1$ th row;

$$
\mathbf{A}_{M+1}=\left[\begin{array}{lllllll}
p_{M+1}-z_{M} & \ldots & p_{M+1}-z_{M} & p_{M+1} & 0 & \ldots & 0
\end{array}\right]
$$

This pattern continues for all category 2 cases up to $M=N-2$, where the final realization is mostly the same but with no 0's left beneath the diagonal;

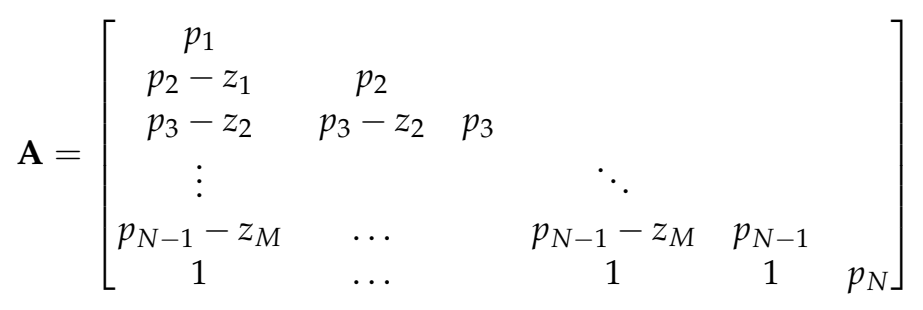


For category 3 where $M=N-1$, there is no room for the row of 1 's, so we construct the A matrix;

$$
\mathbf{A}=\left[\begin{array}{ccccc}
p_{1} & & & & \\
p_{2}-z_{1} & p_{2} & & & \\
p_{3}-z_{2} & p_{3}-z_{2} & p_{3} & & \\
\vdots & & \ddots & \\
p_{N}-z_{M} & \ldots & p_{N}-z_{M} & p_{N}
\end{array}\right]
$$

And thus the row of 1's is shifted to $\mathbf{C}$, as shown above. Now, $\mathbf{C}$ is effectively row $M+2$ which is an important row to consider when constructing the proof. To construct $\mathbf{A}$ for category 4, we follow the same pattern as for category 3 (Equation 15). The difference is then how we must construct $\mathbf{C}$.

\subsection{The special case of $M=N$}

For the case where there are an equal number of poles and zeros, the matrix $\mathbf{D}$ must be nonzero, and in this realization will always be equal to $k$, as shown above. The size of A will always depend on the number of poles; $N \times N$, which is why it is the same as for category 3 (Equation 15).

In order to construct matrix $\mathbf{C}$, each element of the row will now be a summation of pole-zero differences. Be aware these are slightly different to the pole-zero differences in A. By way of example, consider the case of $N=M=1$;

$$
\left[\begin{array}{ll}
\mathbf{A} & \mathbf{B} \\
\mathbf{C} & \mathbf{D}
\end{array}\right]=\left[\begin{array}{cc}
p_{1} & k \\
p_{1}-z_{1} & k
\end{array}\right]
$$

For each $i$ th additional pole and zero, $\mathrm{C}$ expands by 1 column, and $p_{i}-z_{i}$ is added to each element. For instance, for $N=M=2$;

$$
\left[\begin{array}{cc}
\mathbf{A} & \mathbf{B} \\
\mathbf{C} & \mathbf{D}
\end{array}\right]=\left[\begin{array}{ccc}
p_{1} & 0 & k \\
p_{2}-z_{1} & p_{2} & 0 \\
p_{1}-z_{1}+p_{2}-z_{2} & p_{2}-z_{2} & k
\end{array}\right]
$$

or in general, for a function of $N$ poles and zeros;

$$
\mathbf{C}=\left[\begin{array}{llll}
\sum_{i=1}^{N}\left(p_{i}-z_{i}\right) & \ldots & \sum_{i=N-1}^{N}\left(p_{i}-z_{i}\right) & p_{N}-z_{N}
\end{array}\right]
$$

The result of this construction is a series of cancellations when trying to convert the state-space back into a SISO transfer function.

\section{Solving the Laplacian}

Give me the positions and velocities of all the particles in the universe, and I will predict the future.

$$
\text { — Marquis Pierre Simon de Laplace, [13] }
$$

The problem with finding the general solution to these realizations is that the edge cases don't always fit neatly into the regular algebraic patterns. It is easier to work through a number of examples in order to familiarize oneself with the manipulations. These following examples will cover cases from Category 2, then 3 and 4. The Appendix includes a number of simple solutions for quick reference. 
Consider solving the family of differential equations that come from the state-space constructed using Equation 13, such that $M>>0$, and $N>>M$.

$$
\begin{aligned}
\dot{x}_{1} & =p_{1} x_{1}+k u \\
\dot{x}_{2} & =\left(p_{2}-z_{1}\right) x_{1}+p_{2} x_{2} \\
\dot{x}_{3} & =\left(p_{3}-z_{2}\right)\left[x_{1}+x_{2}\right]+p_{3} x_{3} \\
\vdots & \\
\dot{x}_{M+1} & =\left(p_{M+1}-z_{M}\right)\left[x_{1}+\ldots+x_{M}\right]+p_{M+1} x_{M+1} \\
\dot{x}_{M+2} & =\left[x_{1}+\ldots+x_{M}\right]+p_{M+2} x_{M+2} \\
\dot{x}_{M+3} & =x_{M+2}+p_{M+3} x_{M+3} \\
\vdots & \\
\dot{x}_{N} & =x_{N-1}+p_{N} x_{N} \\
y & =x_{N}
\end{aligned}
$$

If we take the Laplace transform of each equation, we get;

$$
\begin{aligned}
X_{1} s & =p_{1} X_{1}+k U \\
X_{2} s & =\left(p_{2}-z_{1}\right) X_{1}+p_{2} X_{2} \\
X_{3} s & =\left(p_{3}-z_{2}\right)\left[X_{1}+X_{2}\right]+p_{3} X_{3} \\
\vdots & \\
X_{M+1} s & =\left(p_{M+1}-z_{M}\right)\left[X_{1}+\ldots+X_{M}\right]+p_{M+1} X_{M+1} \\
X_{M+2} s & =\left[X_{1}+\ldots+X_{M+1}\right]+p_{M+2} X_{M+2} \\
X_{M+3} s & =X_{M+2}+p_{M+3} X_{M+3} \\
\vdots & \\
X_{N} s & =X_{N-1}+p_{N} X_{N} \\
Y & =X_{N}
\end{aligned}
$$

Each equation can then be rearranged to give a specific row by row solution;

$$
\begin{aligned}
X_{1} & =\frac{1}{\left(s-p_{1}\right)} k U \\
X_{2} & =\frac{\left(p_{2}-z_{1}\right)\left[X_{1}\right]}{\left(s-p_{2}\right)} \\
X_{3} & =\frac{\left(p_{3}-z_{2}\right)\left[X_{1}+X_{2}\right]}{\left(s-p_{3}\right)} \\
& \vdots \\
X_{M+1} & =\frac{\left(p_{M+1}-z_{M}\right)\left[X_{1}+\ldots+X_{M}\right]}{\left(s-p_{M+1}\right)} \\
X_{M+2} & =\frac{\left[X_{1}+\ldots+X_{M+1}\right]}{\left(s-p_{M+2}\right)} \\
X_{M+3} & =\frac{X_{M+2}}{\left(s-p_{M+3}\right)} \\
\vdots & \\
X_{N} & =\frac{X_{N-1}}{\left(s-p_{N}\right)} \\
Y & =X_{N}
\end{aligned}
$$


We contend that by solving the $N+1$ equations we will eventually lead back to the regular pole-zero form shown in Equation 1. However, to prove this, we should consider a few simple cases before exploring the full argument, because the patterns are not straightforward. The following proof will proceed through 6 cases;

- $\quad 3.1$ Case 1 - 2 poles, 1 zero

- $\quad 3.2$ Case 2 - 5 poles, 1 zero

- $\quad 3.3$ Case 3 - 5 poles, 3 zeros

- $\quad 3.4$ Case $4-\mathrm{N}$ poles, $\mathrm{M}$ zeros, where $M<N-1$

- $\quad 3.5$ Case $5-\mathrm{N}$ poles, $\mathrm{M}$ zeros, where $M=N-1$

- $\quad 3.6$ Case $6-\mathrm{N}$ poles, $\mathrm{M}$ zeros, where $M=N$

3.1. Case 1 - 2 poles, 1 zero

Firstly, let $N=2$ and $M=1$. Because $M=N-1, \mathbf{C}=[11]$. In this case, the family of 3 equations will be simply;

$$
\begin{aligned}
X_{1} & =\frac{1}{\left(s-p_{1}\right)} k U \\
X_{2} & =\frac{\left(p_{2}-z_{1}\right)\left[X_{1}\right]}{\left(s-p_{2}\right)} \\
Y & =X_{2}+X_{1}
\end{aligned}
$$

Firstly, substituting $X_{1}$ into $X_{2}$;

$$
X_{2}=\frac{\left(p_{2}-z_{1}\right)}{\left(s-p_{2}\right)\left(s-p_{1}\right)} k U
$$

Then $Y$ can be solved easily by substitution;

$$
Y=\frac{\left(p_{2}-z_{1}\right)}{\left(s-p_{2}\right)\left(s-p_{1}\right)} k U+\frac{1}{\left(s-p_{1}\right)} k U
$$

Cross multiply for a common denominator;

$$
Y=\frac{\left(p_{2}-z_{1}\right)}{\left(s-p_{2}\right)\left(s-p_{1}\right)} k U+\frac{\left(s-p_{2}\right)}{\left(s-p_{2}\right)\left(s-p_{1}\right)} k U
$$

Simplify the numerator;

$$
\begin{aligned}
& Y=\frac{\left(p_{2}-z_{1}\right)+\left(s-p_{2}\right)}{\left(s-p_{2}\right)\left(s-p_{1}\right)} k U \\
& Y=\frac{\left(s-z_{1}\right)}{\left(s-p_{2}\right)\left(s-p_{1}\right)} k U
\end{aligned}
$$

This solution matches the form given in equation 1 , and thus works as a proof of that case. What we would like to show is that the poles in the numerator will disappear no matter how many equations. We can show that this cancellation will always occur in the equation for $X_{M+2}$ (except in the case where $M=N-1$, which is shown in Section 3.5, and the case $M=N$, shown in Section 3.6). 
3.2. Case 2 - 5 poles, 1 zero

Consider a second simple case, where $N=5, M=1$, thus $\mathbf{C}=\left[\begin{array}{lllll}0 & 0 & 0 & 0 & 1\end{array}\right]$;

$$
\begin{aligned}
X_{1} & =\frac{1}{\left(s-p_{1}\right)} k U \\
X_{2} & =\frac{\left(p_{2}-z_{1}\right)\left[X_{1}\right]}{\left(s-p_{2}\right)} \\
X_{3} & =\frac{\left[X_{1}+X_{2}\right]}{\left(s-p_{3}\right)} \\
X_{4} & =\frac{X_{3}}{\left(s-p_{4}\right)} \\
X_{5} & =\frac{X_{4}}{\left(s-p_{5}\right)} \\
Y & =X_{5}
\end{aligned}
$$

There are still $N+1$ equations. However, this time note that the last equation to introduce a new zero term is equation $M+1$, and the summation of previous substitutions is in equation for $X_{M+2}$ (i.e. the equation for $X_{3}$ ). The square brackets of this term will be the same as the final solution in Equation 27 (Section 3.1), and therefore;

$$
\begin{aligned}
X_{3} & =\frac{1}{\left(s-p_{3}\right)} \frac{\left(s-z_{1}\right)}{\left(s-p_{2}\right)\left(s-p_{1}\right)} k U \\
X_{4} & =\frac{X_{3}}{\left(s-p_{4}\right)} \\
X_{5} & =\frac{X_{3}}{\left(s-p_{5}\right)} \\
Y & =X_{5}
\end{aligned}
$$

It is trivial to do the substitutions for each line and show that the final solution is

$$
Y=\frac{\left(s-z_{1}\right)}{\left(s-p_{5}\right)\left(s-p_{4}\right)\left(s-p_{3}\right)\left(s-p_{2}\right)\left(s-p_{1}\right)} k U
$$

Which can be written as;

$$
Y=\frac{\left(s-z_{1}\right)}{\prod_{i=1}^{5}\left(s-p_{i}\right)} k U
$$

Once again the solution is in the target form of equation 1. It can also be observed that increasing the number of poles, and thus the number of equations, through $N$ will not make the final substitution any more complicated. Thus, we need to ensure that the first $M+2$ equations can produce the correct numerator for the final solution. 
117 3.3. Case 3 - 5 poles, 3 zeros

Consider the third example case in order to note the pattern of the development of the numerator of $X_{M+2}$. Consider $N=5, M=3$, thus $\mathbf{C}=\left[\begin{array}{lllll}0 & 0 & 0 & 0 & 1\end{array}\right]$;

$$
\begin{aligned}
X_{1} & =\frac{1}{\left(s-p_{1}\right)} k U \\
X_{2} & =\frac{\left(p_{2}-z_{1}\right)}{\left(s-p_{2}\right)}\left[X_{1}\right] \\
X_{3} & =\frac{\left(p_{3}-z_{2}\right)}{\left(s-p_{3}\right)}\left[X_{1}+X_{2}\right] \\
X_{4} & =\frac{\left(p_{4}-z_{3}\right)}{\left(s-p_{4}\right)}\left[X_{1}+X_{2}+X_{3}\right] \\
X_{5} & =\frac{1}{\left(s-p_{5}\right)}\left[X_{1}+X_{2}+X_{3}+X_{4}\right] \\
Y & =X_{5}
\end{aligned}
$$

Taking what we know from previous examples, $X_{2}$ simplifies to;

$$
X_{2}=\frac{\left(p_{2}-z_{1}\right)}{\left(s-p_{2}\right)\left(s-p_{1}\right)} k U
$$

We know $\left[X_{1}+X_{2}\right]$ from Equation 27, thus $X_{3}$;

$$
\begin{gathered}
X_{3}=\frac{\left(p_{3}-z_{2}\right)}{\left(s-p_{3}\right)}\left[\frac{\left(s-z_{1}\right)}{\left(s-p_{2}\right)\left(s-p_{1}\right)} k U\right] \\
X_{3}=\frac{\left(p_{3}-z_{2}\right)\left(s-z_{1}\right)}{\left(s-p_{2}\right)\left(s-p_{1}\right)\left(s-p_{3}\right)} k U
\end{gathered}
$$

Consider $X_{4}$;

$$
\begin{aligned}
X_{4}= & \frac{\left(p_{4}-z_{3}\right)}{\left(s-p_{4}\right)} \times\left[\frac{1}{\left(s-p_{1}\right)} k U+\right. \\
& \frac{\left(p_{2}-z_{1}\right)}{\left(s-p_{2}\right)\left(s-p_{1}\right)} k U+ \\
& \left.\frac{\left(p_{3}-z_{2}\right)\left(s-z_{1}\right)}{\left(s-p_{2}\right)\left(s-p_{1}\right)\left(s-p_{3}\right)} k U\right]
\end{aligned}
$$

Create the common denominator of the square brackets;

$$
\begin{aligned}
X_{4}= & \frac{\left(p_{4}-z_{3}\right)}{\left(s-p_{4}\right)} \times\left[\frac{\left(s-p_{3}\right)\left(s-p_{2}\right)}{\left(s-p_{3}\right)\left(s-p_{2}\right)\left(s-p_{1}\right)} k U+\right. \\
& \frac{\left(s-p_{3}\right)\left(p_{2}-z_{1}\right)}{\left(s-p_{3}\right)\left(s-p_{2}\right)\left(s-p_{1}\right)} k U+ \\
& \left.\frac{\left(p_{3}-z_{2}\right)\left(s-z_{1}\right)}{\left(s-p_{2}\right)\left(s-p_{1}\right)\left(s-p_{3}\right)} k U\right]
\end{aligned}
$$

Factorize;

$$
\begin{aligned}
X_{4}= & \frac{\left(p_{4}-z_{3}\right)}{\prod_{j=1}^{4}\left(s-p_{j}\right)} k U \times\left[\left(s-p_{3}\right)\left(s-p_{2}\right)+\right. \\
& \left.\left(s-p_{3}\right)\left(p_{2}-z_{1}\right)+\left(p_{3}-z_{2}\right)\left(s-z_{1}\right)\right]
\end{aligned}
$$


Simplifying the square brackets is trivial, but we are interested in establishing a pattern to solve the general case. Consider that there are three terms within the square brackets. Notice that only the first two contain combinations of $p_{2}$. We can eliminate that first. Start by expanding only the brackets that contain $p_{2}$;

$$
\begin{array}{r}
{\left[\left(s-p_{3}\right) s-\left(s-p_{3}\right) p_{2}+\left(s-p_{3}\right) p_{2}-\left(s-p_{3}\right) z_{1}+\right.} \\
\left.\left(p_{3}-z_{2}\right)\left(s-z_{1}\right)\right]
\end{array}
$$

All of the $p_{2}$ terms cancel, and the remaining $\left(s-p_{3}\right)$ terms can factorize;

$$
\left[\left(s-p_{3}\right)\left(s-z_{1}\right)+\left(p_{3}-z_{2}\right)\left(s-z_{1}\right)\right]
$$

Repeating the process by expanded the brackets containing $p_{3}$, cancelling, and refactorising, the square brackets reduce to;

$$
\left[\left(s-z_{2}\right)\left(s-z_{1}\right)\right]
$$

And therefore;

$$
X_{4}=\frac{\left(p_{4}-z_{3}\right)\left(s-z_{2}\right)\left(s-z_{1}\right)}{\prod_{j=1}^{4}\left(s-p_{j}\right)} k U
$$

In this case, we are most interested in the equation for $X_{5}$, which is the $X_{M+2}$ equation. If we write in out in full, finding the common denominator of the square brackets as we did above, we get;

$$
\begin{aligned}
X_{5}= & \frac{1}{\prod_{j=1}^{5}\left(s-p_{j}\right)} k U \times \ldots \\
& {\left[\left(s-p_{3}\right)\left(s-p_{2}\right)\left(s-p_{1}\right)+\right.} \\
& \left(p_{2}-z_{1}\right)\left(s-p_{4}\right)\left(s-p_{3}\right)+ \\
& \left(p_{3}-z_{2}\right)\left(s-p_{4}\right)\left(s-z_{1}\right)+ \\
& \left.\left(p_{4}-z_{3}\right)\left(s-z_{2}\right)\left(s-z_{1}\right)\right]
\end{aligned}
$$

From this step, we can simplify the square brackets by expanding, cancelling, and refactorising as we did in Equations 39 and 40. This would give us the final solution in the form;

$$
Y=\frac{\prod_{i=1}^{3}\left(s-z_{i}\right)}{\prod_{j=1}^{5}\left(s-p_{i}\right)} \cdot k U
$$

More importantly,we can observe that finally a pattern is emerging for finding a 
3.4. Case 4 - $N$ poles, $M$ zeros, where $M<N-1$

Using equation 43 as a base, we can generalise the equation for $X_{M+2}$ to;

$$
\begin{aligned}
X_{M+2}= & \frac{k U}{\prod_{j=1}^{M+2}\left(s-p_{j}\right)} \times\left[\prod_{i=2}^{M+1}\left(s-p_{i}\right)+\right. \\
& \left(p_{2}-z_{1}\right) \prod_{i=3}^{M+1}\left(s-p_{i}\right)+ \\
& \left(p_{3}-z_{2}\right) \prod_{i=4}^{M+1}\left(s-p_{i}\right) \cdot\left(s-z_{1}\right)+ \\
& \left(p_{4}-z_{3}\right) \prod_{i=5}^{M+1}\left(s-p_{i}\right) \cdot \prod_{i=1}^{2}\left(s-z_{i}\right)+\ldots+ \\
& \left(p_{M}-z_{M-1}\right)\left(s-p_{M+1}\right) \cdot \prod_{i=1}^{M-2}\left(s-z_{i}\right)+ \\
& \left.\left(p_{M+1}-z_{M}\right) \prod_{i=1}^{M-1}\left(s-z_{i}\right)\right]
\end{aligned}
$$

We can simplify the square brackets using the techniques used in Equations 39 and 40 above. As an example, consider just the first two terms;

$$
\left[\prod_{i=2}^{M+1}\left(s-p_{i}\right)+\left(p_{2}-z_{1}\right) \prod_{i=3}^{M+1}\left(s-p_{i}\right)+\ldots\right]
$$

And rearrange the first term to show $\left(s-p_{2}\right)$ explicitly;

$$
\left[\left(s-p_{2}\right) \prod_{i=3}^{M+1}\left(s-p_{i}\right)+\left(p_{2}-z_{1}\right) \prod_{i=3}^{M+1}\left(s-p_{i}\right)+\ldots\right]
$$

Expand the brackets containing $p_{2}$;

$$
\begin{array}{r}
{\left[(s) \prod_{i=3}^{M+1}\left(s-p_{i}\right)-\left(p_{2}\right) \prod_{i=3}^{M+1}\left(s-p_{i}\right)+\right.} \\
\left.\left(p_{2}\right) \prod_{i=3}^{M+1}\left(s-p_{i}\right)-\left(z_{1}\right) \prod_{i=3}^{M+1}\left(s-p_{i}\right)+\ldots\right]
\end{array}
$$

All the terms with $p_{2}$ cancel, and the rest of the terms can be factorized;

$$
\left[\prod_{i=3}^{M+1}\left(s-p_{i}\right) \cdot\left(s-z_{1}\right)+\ldots\right]
$$

If we factor out the term $\left(s-p_{3}\right)$, and consider one more term from within the continuation, we get;

$$
\begin{array}{r}
{\left[\left(s-p_{3}\right) \prod_{i=4}^{M+1}\left(s-p_{i}\right) \cdot\left(s-z_{1}\right)+\right.} \\
\left.\left(p_{3}-z_{2}\right) \prod_{i=4}^{M+1}\left(s-p_{i}\right) \cdot\left(s-z_{1}\right)+\ldots\right]
\end{array}
$$


This is similar to what we see in Equation 47, and thus we can repeat the steps leading up all the way to the final two;

$$
\left[\left(s-p_{M+1}\right) \prod_{i=1}^{M-1}\left(s-z_{i}\right)+\left(p_{M+1}-z_{M}\right) \prod_{i=1}^{M-1}\left(s-z_{i}\right)\right]
$$

Which expands and reduces to

$$
\prod_{i=1}^{M}\left(s-z_{i}\right)
$$

Finally, consider the full equation for $X_{M+2}$;

$$
X_{M+2}=\frac{\prod_{i=1}^{M}\left(s-z_{i}\right)}{\prod_{j=1}^{M+2}\left(s-p_{j}\right)} k U
$$

Further terms, such that;

$$
\begin{aligned}
X_{M+2} & =\frac{\prod_{i=1}^{M}\left(s-z_{i}\right)}{\prod_{j=1}^{M+2}\left(s-p_{j}\right)} k U \\
X_{M+3} & =\frac{X_{M+2}}{\left(s-p_{M+3}\right)} \\
\vdots & \\
X_{N} & =\frac{X_{N-1}}{\left(s-p_{N}\right)} \\
Y & =X_{N}
\end{aligned}
$$

or, in the case that $M=N-2$, then;

$$
Y=X_{M+2}=X_{N}
$$

Either way, the solution is trivially;

$$
Y=\frac{\prod_{i=1}^{M}\left(s-z_{i}\right)}{\prod_{j=1}^{N}\left(s-p_{i}\right)} k U(s)
$$

122 3.5. Case 5 - $N$ poles, $M$ zeros, where $M=N-1$

For category 3 functions, the procedure is slightly different from the above but follows much the same logic. Consider that we solve all but the final 2 equations in the Laplace system of equations;

$$
\begin{aligned}
X_{N} & =\frac{\left(p_{N}-z_{N-1}\right)}{\left(s-p_{N}\right)}\left[X_{1}+X_{2}+\ldots+X_{N-1}\right] \\
Y & =\left[X_{1}+X_{2}+\ldots+X_{N}\right]
\end{aligned}
$$


Following the patterns we explored previously; the solution to this set of equations is;

$$
\begin{aligned}
Y= & \frac{k U}{\prod_{j=1}^{N}\left(s-p_{j}\right)} \times\left[\prod_{i=2}^{N}\left(s-p_{i}\right)+\right. \\
& \left(p_{2}-z_{1}\right) \prod_{i=3}^{N}\left(s-p_{i}\right)+ \\
& \left(p_{3}-z_{2}\right) \prod_{i=4}^{N}\left(s-p_{i}\right) \cdot\left(s-z_{1}\right)+ \\
& \left(p_{4}-z_{3}\right) \prod_{i=5}^{N}\left(s-p_{i}\right) \cdot \prod_{i=1}^{2}\left(s-z_{i}\right)+\ldots+ \\
& \left(p_{N-1}-z_{N-2}\right)\left(s-p_{N}\right) \cdot \prod_{i=1}^{N-3}\left(s-z_{i}\right)+ \\
& \left.\left(p_{N}-z_{N-1}\right) \prod_{i=1}^{N-2}\left(s-z_{i}\right)\right]
\end{aligned}
$$

There is one less term in the denominator then previously expected, because there is no denominator for $Y$. The square brackets simply reduce down to;

$$
Y=\frac{k U}{\prod_{j=1}^{N}\left(s-p_{j}\right)} \times\left[\prod_{i=1}^{N-1}\left(s-z_{i}\right)\right]
$$

Which is equivalent to

$$
Y=\frac{\prod_{i=1}^{M}\left(s-z_{i}\right)}{\prod_{j=1}^{N}\left(s-p_{i}\right)} k U(s)
$$

3.6. Case $6-N$ poles, $M$ zeros, where $M=N$

The final case, adds a level of complexity to the algebraic operations, but the result is very similar. Again, consider all but the final 2 equations in the Laplace system of equations as above, but before we have inserted the correct formulation of $\mathbf{C}$ and $\mathbf{D}$;

$$
\begin{array}{r}
X_{N}=\frac{\left(p_{N}-z_{N-1}\right)}{\left(s-p_{N}\right)}\left[X_{1}+X_{2}+\ldots+X_{N-1}\right] \\
Y=\left[\mathbf{C}_{1} X_{1}+\mathbf{C}_{2} X_{2}+\ldots+\mathbf{C}_{N} X_{N}\right]+\mathbf{D} U
\end{array}
$$

The formula for $Y$ is similar to Case 5 , but with a single additional term $\mathbf{D}=\mathrm{k}$. Start by rearranging the $\mathbf{D}$ to the front, and substituting in all the components of $\mathbf{C}$ (also note, for clarity, more elements are shown and the $\mathbf{C}$ terms are in angle brackets " \langle\rangle ");

$$
\begin{gathered}
Y=k U+X_{1}\left\langle\sum_{i=1}^{N}\left(p_{i}-z_{i}\right)\right\rangle+X_{2}\left\langle\sum_{i=2}^{N}\left(p_{i}-z_{i}\right)\right\rangle+ \\
X_{3}\left\langle\sum_{i=3}^{N}\left(p_{i}-z_{i}\right)\right\rangle+X_{4}\left\langle\sum_{i=4}^{N}\left(p_{i}-z_{i}\right)\right\rangle+\ldots+ \\
\left.X_{N-1}\left\langle\sum_{i=N-1}^{N}\left(p_{i}-z_{i}\right)\right\rangle+X_{N}\left\langle p_{N}-z_{N}\right)\right\rangle
\end{gathered}
$$


Now substitute in the solutions for all $X_{i}$, recognise there is a common factor $\mathrm{k} U$, and multiply through to create the common denominator; $Y=$

$$
\begin{aligned}
& \frac{k U}{\prod_{j=1}^{N}\left(s-p_{j}\right)} \times\left[\prod_{i=1}^{N}\left(s-p_{i}\right)+\right. \\
& \prod_{i=2}^{N}\left(s-p_{i}\right)\left\langle\sum_{i=1}^{N}\left(p_{i}-z_{i}\right)\right\rangle+ \\
& \left(p_{2}-z_{1}\right) \prod_{i=3}^{N}\left(s-p_{i}\right)\left\langle\sum_{i=2}^{N}\left(p_{i}-z_{i}\right)\right\rangle+ \\
& \left(p_{3}-z_{2}\right) \prod_{i=4}^{N}\left(s-p_{i}\right) \cdot\left(s-z_{1}\right)\left\langle\sum_{i=3}^{N}\left(p_{i}-z_{i}\right)\right\rangle+ \\
& \left(p_{4}-z_{3}\right) \prod_{i=5}^{N}\left(s-p_{i}\right) \cdot \prod_{i=1}^{2}\left(s-z_{i}\right)\left\langle\sum_{i=4}^{N}\left(p_{i}-z_{i}\right)\right\rangle+\ldots+ \\
& \left(p_{N-1}-z_{N-2}\right)\left(s-p_{N}\right) \cdot \prod_{i=1}^{N-3}\left(s-z_{i}\right)\left\langle\sum_{i=N-1}^{N}\left(p_{i}-z_{i}\right)\right\rangle+ \\
& \left.\left(p_{N}-z_{N-1}\right) \prod_{i=1}^{N-2}\left(s-z_{i}\right)\left\langle p_{N}-z_{N}\right\rangle\right]
\end{aligned}
$$

This reduces to

$$
\begin{aligned}
& {\left[\left(s-z_{1}\right) \prod_{i=2}^{N}\left(s-p_{i}\right)+\right.} \\
& \left.\prod_{i=2}^{N}\left(s-p_{i}\right)\left\langle\sum_{i=2}^{N}\left(p_{i}-z_{i}\right)\right\rangle \ldots\right]
\end{aligned}
$$

131

132

$$
\begin{aligned}
& {\left[(s) \prod_{i=2}^{N}\left(s-p_{i}\right)-\left(p_{1}\right) \prod_{i=2}^{N}\left(s-p_{i}\right)+\right.} \\
& \left(p_{1}\right) \prod_{i=2}^{N}\left(s-p_{i}\right)-\left(z_{1}\right) \prod_{i=2}^{N}\left(s-p_{i}\right)+ \\
& \left.\prod_{i=2}^{N}\left(s-p_{i}\right)\left\langle\sum_{i=2}^{N}\left(p_{i}-z_{i}\right)\right\rangle \ldots\right]
\end{aligned}
$$

This represents the new first and second terms of the square brackets. Eliminating $p_{2}$ is done in two steps, as does the rest of the $p$ terms. Consider the second and third term of the square brackets, with $\left(s-p_{2}\right)$ brought to the front ;

$$
\begin{aligned}
& {\left[\ldots\left(s-p_{2}\right) \prod_{i=3}^{N}\left(s-p_{i}\right)\left\langle\sum_{i=2}^{N}\left(p_{i}-z_{i}\right)\right\rangle+\right.} \\
& \left.\left(p_{2}-z_{1}\right) \prod_{i=3}^{N}\left(s-p_{i}\right)\left\langle\sum_{i=2}^{N}\left(p_{i}-z_{i}\right)\right\rangle \ldots\right]
\end{aligned}
$$


We can expand the foremost brackets for $p_{2}$, which then cancel, and these terms simplify to;

$$
\left[\ldots\left(s-z_{1}\right) \prod_{i=3}^{N}\left(s-p_{i}\right)\left\langle\sum_{i=2}^{N}\left(p_{i}-z_{i}\right)\right\rangle \ldots\right]
$$

And now we can compare this with the first term

$$
\begin{aligned}
& {\left[\left(s-z_{1}\right) \prod_{i=2}^{N}\left(s-p_{i}\right)+\right.} \\
& \left.\left(s-z_{1}\right) \prod_{i=3}^{N}\left(s-p_{i}\right)\left\langle\sum_{i=2}^{N}\left(p_{i}-z_{i}\right)\right\rangle \ldots\right]
\end{aligned}
$$

Expand for the remaining $p_{2}$ terms, and $z_{2}$ terms in the angle brackets;

$$
\begin{aligned}
& {\left[(s)\left(s-z_{1}\right) \prod_{i=3}^{N}\left(s-p_{i}\right)-\left(p_{2}\right)\left(s-z_{1}\right) \prod_{i=3}^{N}\left(s-p_{i}\right)+\right.} \\
& \left(p_{2}\right)\left(s-z_{1}\right) \prod_{i=3}^{N}\left(s-p_{i}\right)-\left(z_{2}\right)\left(s-z_{1}\right) \prod_{i=3}^{N}\left(s-p_{i}\right)+ \\
& \left.\left(s-z_{1}\right) \prod_{i=3}^{N}\left(s-p_{i}\right)\left\langle\sum_{i=3}^{N}\left(p_{i}-z_{i}\right)\right\rangle \ldots\right]
\end{aligned}
$$

All the $p_{2}$ can now be eliminated;

$$
\begin{aligned}
& {\left[\prod_{i=1}^{2}\left(s-z_{i}\right) \prod_{i=3}^{N}\left(s-p_{i}\right)+\right.} \\
& \left.\left(s-z_{1}\right) \prod_{i=3}^{N}\left(s-p_{i}\right)\left\langle\sum_{i=3}^{N}\left(p_{i}-z_{i}\right)\right\rangle \ldots\right]
\end{aligned}
$$

This two step pattern of eliminating $p$ terms can be repeated until all of them are eliminated, resulting in the square brackets equal to;

$$
\left[\prod_{i=1}^{N}\left(s-z_{i}\right)\right]
$$

And thus it is finally found that

$$
Y=\frac{\prod_{i=1}^{N}\left(s-z_{i}\right)}{\prod_{j=1}^{N}\left(s-p_{j}\right)} k U(s)
$$

\section{Comparison with other Canonical Forms}

In most fields, a canonical form specifies a unique representation for every object, while a normal form simply specifies its form, without the requirement of uniqueness.

4.1. Category 3. SISO Transfer Functions with $n$ poles and $n-1$ zeros

$$
\frac{Y(s)}{U(s)}=\frac{b_{1} s^{n-1}+\ldots+b_{n-1} s+b_{n}}{s^{n}+a_{1} s^{n-1}+\ldots+a_{n-1} s+a_{n}}
$$




\begin{tabular}{rcc}
\hline \multicolumn{1}{l}{ Controllable Canonical Form: } & \\
$\mathbf{A}=\left[\begin{array}{ccccc}0 & 1 & & 0 & 0 \\
\vdots & & \ddots & & \vdots \\
0 & 0 & & 1 & 0 \\
0 & 0 & \ldots & 0 & 1 \\
-a_{n} & -a_{n-1} & \ldots & -a_{2} & -a_{1}\end{array}\right]$ & $\mathbf{B}=\left[\begin{array}{c}0 \\
\vdots \\
0 \\
0 \\
1\end{array}\right]$ \\
$\mathbf{C}=\left[\begin{array}{lllll}b_{n} & b_{n-1} & \ldots & b_{2} & b_{1}\end{array}\right]$ & $\mathbf{D}=[0]$
\end{tabular}

\begin{aligned} & \hline \multicolumn{3}{l}{ Observable Canonical Form: } \\ & $\mathbf{A}=\left[\begin{array}{ccccc}0 & \ldots & 0 & 0 & -a_{n} \\ 1 & & 0 & 0 & -a_{n-1} \\ & \ddots & & \vdots & \vdots \\ 0 & & 1 & 0 & -a_{2} \\ 0 & \ldots & 0 & 1 & -a_{1}\end{array}\right] \mathbf{B}=\left[\begin{array}{c}b_{n} \\ b_{n-1} \\ \vdots \\ b_{2} \\ b_{1}\end{array}\right] \\ & \mathbf{C}=\left[\begin{array}{llllc|}0 & \ldots & 0 & 0 & 1\end{array}\right] \mathbf{D}=[0] \\ &$\hline\end{aligned}

Diagonal Canonical Form: Factorize denominator, compute partial fractions;

$$
\begin{array}{rlrl}
\frac{Y(s)}{U(s)} & =\frac{b_{1} s^{n-1}+\ldots+b_{n-1} s+b_{n}}{\left(s-p_{1}\right)\left(s-p_{2}\right) \ldots\left(s-p_{n}\right)} & \\
& =\frac{r_{1}}{\left(s-p_{1}\right)}+\frac{r_{2}}{\left(s-p_{2}\right)}+\ldots+\frac{r_{n}}{\left(s-p_{n}\right)} \\
\mathbf{A} & =\left[\begin{array}{ccccc}
p_{1} & 0 & \ldots & 0 & 0 \\
0 & p_{2} & & 0 & 0 \\
\vdots & & \ddots & & \vdots \\
0 & 0 & & p_{n-1} & 0 \\
0 & 0 & \ldots & 0 & p_{n}
\end{array}\right] \quad \mathbf{B}=\left[\begin{array}{c}
r_{1} \\
r_{2} \\
\vdots \\
r_{n-1} \\
r_{n}
\end{array}\right] \\
\mathbf{C}=\left[\begin{array}{lllll}
1 & 1 & \ldots & 1 & 1
\end{array}\right] & \mathbf{D}=[0]
\end{array}
$$

Pole-Zero Difference Form: Factorize the numerator and denominator;

$$
\begin{aligned}
& \frac{Y(s)}{U(s)}=\frac{\left(s-z_{1}\right)\left(s-z_{2}\right) \ldots\left(s-z_{n-1}\right)}{\left(s-p_{1}\right)\left(s-p_{2}\right) \ldots\left(s-p_{n}\right)} k \\
& \begin{array}{rlcccc}
\mathbf{A} & =\left[\begin{array}{ccccc}
p_{1} & 0 & 0 & & 0 \\
\Delta_{21} & p_{2} & 0 & \ldots & 0 \\
\Delta_{32} & \Delta_{32} & p_{3} & & 0 \\
\vdots & & & \ddots & \\
\Delta_{n, n-1} & \Delta_{n, n-1} & \Delta_{n, n-1} & \ldots & p_{n}
\end{array}\right] & \mathbf{B}=\left[\begin{array}{c}
k \\
0 \\
\vdots \\
0 \\
0
\end{array}\right] \\
\mathbf{C}=\left[\begin{array}{lllll}
1 & 1 & 1 & \ldots & 1
\end{array}\right] & & \mathbf{D}=[0]
\end{array} \\
& \text { where } \Delta_{i j}=p_{i}-z_{j}
\end{aligned}
$$

4.2. Category 4. SISO Transfer Functions with $n$ poles and $n$ zeros

$$
\frac{Y(s)}{U(s)}=\frac{b_{0} s^{n}+b_{1} s^{n-1}+\ldots+b_{n-1} s+b_{n}}{s^{n}+a_{1} s^{n-1}+\ldots+a_{n-1} s+a_{n}}
$$




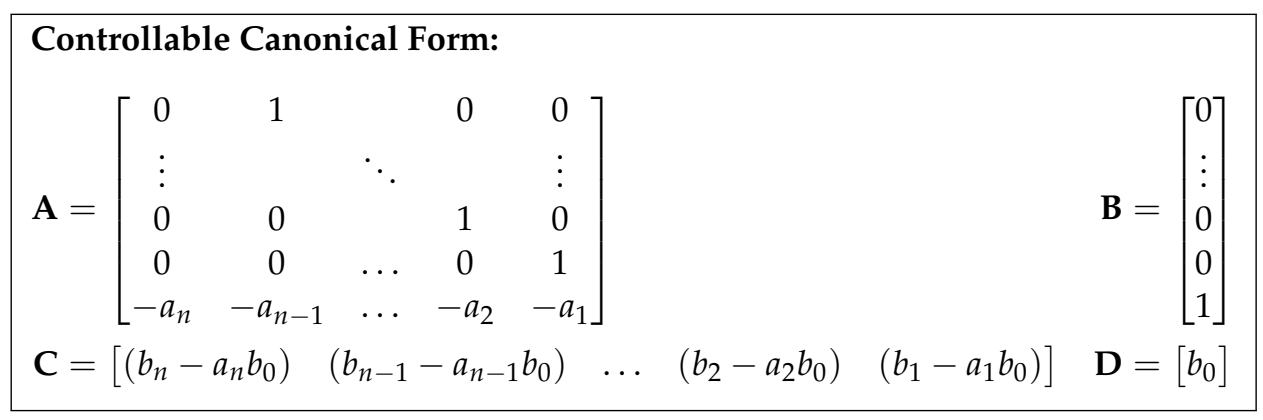

\section{Observable Canonical Form:}

$$
\begin{aligned}
\mathbf{A}=\left[\begin{array}{ccccc}
0 & \ldots & 0 & 0 & -a_{n} \\
1 & & 0 & 0 & -a_{n-1} \\
& \ddots & & \vdots & \vdots \\
0 & & 1 & 0 & -a_{2} \\
0 & \ldots & 0 & 1 & -a_{1}
\end{array}\right] & \mathbf{B}=\left[\begin{array}{c}
b_{n}-a_{n} b_{0} \\
b_{n-1}-a_{n-1} b_{0} \\
\vdots \\
b_{2}-a_{2} b_{0} \\
b_{1}-a_{1} b_{0}
\end{array}\right] \\
\mathbf{C}=\left[\begin{array}{llllc}
0 & \ldots & 0 & 0 & 1
\end{array}\right] & \mathbf{D}=\left[b_{0}\right]
\end{aligned}
$$

Diagonal Canonical Form: Factorize denominator, compute partial fractions;

$$
\begin{array}{ccc}
\frac{Y(s)}{U(s)} & =\frac{b_{0} s^{n}+b_{1} s^{n-1}+\ldots+b_{n-1} s+b_{n}}{\left(s-p_{1}\right)\left(s-p_{2}\right) \ldots\left(s-p_{n}\right)} \\
& =b_{0}+\frac{r_{1}}{\left(s-p_{1}\right)}+\frac{r_{2}}{\left(s-p_{2}\right)}+\ldots+\frac{r_{n}}{\left(s-p_{n}\right)} \\
\mathbf{A} & =\left[\begin{array}{ccccc}
p_{1} & 0 & \ldots & 0 & 0 \\
0 & p_{2} & & 0 & 0 \\
\vdots & & \ddots & & \vdots \\
0 & 0 & & p_{n-1} & 0 \\
0 & 0 & \ldots & 0 & p_{n}
\end{array}\right] \quad \mathbf{B}=\left[\begin{array}{c}
r_{1} \\
r_{2} \\
\vdots \\
r_{n-1} \\
r_{n}
\end{array}\right] \\
\mathbf{C}=\left[\begin{array}{lllll}
1 & 1 & \ldots & 1 & 1
\end{array}\right] \quad \mathbf{D}=\left[b_{0}\right]
\end{array}
$$

Pole-Zero Difference Form: Factorize the numerator and denominator;

$$
\frac{Y(s)}{U(s)}=\frac{\left(s-z_{1}\right)\left(s-z_{2}\right) \ldots\left(s-z_{n}\right)}{\left(s-p_{1}\right)\left(s-p_{2}\right) \ldots\left(s-p_{n}\right)} k
$$

$$
\begin{array}{llllll}
\mathbf{A} & =\left[\begin{array}{ccccc}
p_{1} & 0 & 0 & & 0 \\
\Delta_{21} & p_{2} & 0 & \ldots & 0 \\
\Delta_{32} & \Delta_{32} & p_{3} & & 0 \\
\vdots & & & \ddots & \\
\Delta_{n, n-1} & \Delta_{n, n-1} & \Delta_{n, n-1} & \ldots & p_{n}
\end{array}\right] & \mathbf{B}=\left[\begin{array}{c}
k \\
0 \\
\vdots \\
0 \\
0
\end{array}\right] \\
\mathbf{C}=\left[\begin{array}{lllll}
\left(\sum_{i=1}^{n} \Delta_{i i}\right) & \left(\sum_{i=2}^{n} \Delta_{i i}\right) & \ldots & \left(\Delta_{n-1, n-1}+\Delta_{n n}\right) & \left(\Delta_{n n}\right)
\end{array}\right] & \mathbf{D}=[k]
\end{array}
$$

where $\Delta_{i j}=p_{i}-z_{j}$

\section{Discussion}

Mathematicians seem to have no difficulty in creating new concepts faster than the old ones become well understood. 
Acceptance Speech for the 1991 Kyoto Prize, 'A scientist by choice'. On kyotoprize.org website. [15]

We propose a method to construct a state-space realization such that the numerical values of the poles, zeros and gain appear explicitly in the standard state space matrices, and designate it Pole-Zero-Difference form, where the name stems from the off-diagonal terms in the A matrix of the standard state space form. Realizations in this form have been found for all proper transfer functions and will be presented here. We demonstrated that by solving the $\mathrm{N}+1$ equations we will eventually lead back to the regular pole-zero form. Poles, zeros and gain may now be easily extracted an applied broadly to systems across the applied sciences expressed in state space form.

\section{Author Contributions:}

Conceptualization, A.V.; methodology, A.V.; validation, A.V.; formal analysis, A.V.; investigation, A.V.; resources, T.S.; writing-original draft preparation, A.V.; writing-review and editing, A.V. and T.S.; project administration, T.S.; funding acquisition, T.S. All authors have read and agreed to the published version of the manuscript. Please turn to the CRediT taxonomy for the term explanation. Authorship must be limited to those who have contributed substantially to the work reported.

Funding: This research received no external funding.

\section{References}

1. Lorenz, E. Introducing Chaos Theory in relation to weather prediction, Deterministic Nonperiodic Flow. J. Atm. Sci., 1963, 20, 130.

2. Wingett, P.; Sharon, K.; Brault, C.; Potter, C. Unmanned underwater vehicle turbine powered charging system and method. US Patent US7077072B2, July 18, 2006.

3. van Paridon, A.; Bacic, M.; Ireland, P.; Daniel, R.; On the Real-Time Estimation of Disk Temperature Spatial Distributions in Aeroengines. J. Eng. Gas Turbines Power 2018, 140(3), 031901.

4. Canuto, E.; Novara, C.; Massotti, L.; Carlucci, D.; Montenegro, C. Chapter 1 - Introduction. In Aerospace Engineering, Spacecraft Dynamics and Control, 2nd ed.; Canuto, E.; Novara, C.; Massotti, L.; Carlucci, D.; Butterworth-Heinemann: Oxford, United Kingdom, 2018; pp. 1-16. https://doi.org/10.1016/B978-0-08-100700-6.00001-5

5. Singh, A.; Pal, B.; Centralized Dynamic Estimation and Control. Dynamic Estimation and Control of Power Systems; Elsevier Academic Press: Cambridge, USA, 2019; pp. 35-60. https://doi.org/10.1016/B978-0-12-814005-5.00014-5

6. Svinin, M. Goncharenko, I; Kryssanov, V.; Yamamoto, M; Chapter 5 - Modeling and Human Performance in Manipulating Parallel Flexible Objects. In Human Inspired Dexterity in Robotic Manipulation, Watanabe, T.; Harada, K.; Tada, M.; Elsevier Academic Press: Cambridge, USA, 2019; pp. 61-83. https://doi.org/10.1016/B978-0-12-813385-9.00005-4

7. Nenchev, D.; Konno, A.; Tsujita, T.; Chapter 5 - Balance Control, In Humanoid Robots; enchev, D.; Konno, A.; Tsujita, T.; Butterworth-Heinemann: Oxford, United Kingdom, 2019; pp. 203-302. https://doi.org/10.1016/B978-0-12-804560-2.00012-2.

8. Canuto, E.; Novara, C.; Massotti, L.; Carlucci, D.; Montenegro, C. Chapter 2 - Attitude Representation. In Aerospace Engineering, Spacecraft Dynamics and Control, 2nd ed.; Canuto, E.; Novara, C.; Massotti, L.; Carlucci, D.; Butterworth-Heinemann: Oxford, United Kingdom, 2018; pp. 17-83. https://doi.org/10.1016/B978-0-08-100700-6.00001-5

9. Mondal, D.; Chakrabarti, A.; Sengupta, A.; Chapter 4 - Small-Signal Stability Analysis in SMIB Power System. In Power System Small Signal Stability Analysis and Control, 2nd ed.; Mondal, D.; Chakrabarti, A.; Sengupta; A.; Elsevier Academic Press: Cambridge, USA, 2020; pp. 87-123. https://doi.org/10.1016/B978-0-12-813385-9.00005-4 
10. Kaipio, J; Rimpiläinen, V.; Process tomography and estimation of velocity fields. In Woodhead Publishing Series in Electronic and Optical Materials, Industrial Tomography; Wang, M.; Woodhead Publishing: Cambridge, United Kingdom, 2015; pp. 551-590. https://doi.org/10.1016/B9781-78242-118-4.00021-6.

11. Dixon, A.; Chapter 8 - The "control diagram" method. In Aspects of Power System Frequency Stability and Control; Dixon, A.; Elsevier Academic Press: Cambridge, USA, 2019; pp. 191209. https:/ / doi.org/10.1016/B978-0-12-816139-5.00008-4

12. Palmer, T. Edward Norton Lorenz: 23 May 1917-16 April 2008. Biographical Memoirs of Fellows of the Royal Society 2009, 55, 144.

13. Laplace, P. A philosophical essay on probabilities; Wiley: New York, USA, 1902. Available online: https://archive.org/details/philosophicaless00lapliala/page/2/mode/2up (accessed on 2 Feb 2022). As quoted in Agar, J. Turing and the Universal Machine. Icon Books: London, U.K., 2001. Available online: https://books.google.com/books?id=q9wnDwAAQBAJpg=PT53lpg=PT

14. Shilov, G.; Silverman, R. (ed.), Linear Algebra, Dover: Mineola, USA, 1977. ISBN 0-486-63518X.

15. 1991 Kyoto Prize Laureates. Available online: https://www.kyotoprize.org/en/laureates/edward_no (accessed on 2 Feb 2022).

\section{APPENDIX}

Here the reader can find examples of the conversion for some simple cases, as a quick reference tool.

1. Transfer Function with 1 Pole, 0 Zeros

$$
\begin{gathered}
\frac{Y(s)}{U(s)}=\frac{1}{\left(s-p_{1}\right)} k \\
{\left[\begin{array}{cc}
\mathbf{A} & \mathbf{B} \\
\mathbf{C} & \mathbf{D}
\end{array}\right]=\left[\begin{array}{cc}
p_{1} & k \\
1 & 0
\end{array}\right]}
\end{gathered}
$$

2. Transfer Function with 1 Pole, 1 Zero

$$
\begin{gathered}
\frac{Y(s)}{U(s)}=\frac{\left(s-z_{1}\right)}{\left(s-p_{1}\right)} k \\
{\left[\begin{array}{ll}
\mathbf{A} & \mathbf{B} \\
\mathbf{C} & \mathbf{D}
\end{array}\right]=\left[\begin{array}{cc}
p_{1} & k \\
p_{1}-z_{1} & k
\end{array}\right]}
\end{gathered}
$$

3. Transfer Function with 2 Poles 0 Zeros

$$
\begin{aligned}
& \frac{Y(s)}{U(s)}=\frac{1}{\left(s-p_{1}\right)\left(s-p_{2}\right)} k \\
& {\left[\begin{array}{ll}
\mathbf{A} & \mathbf{B} \\
\mathbf{C} & \mathbf{D}
\end{array}\right]=\left[\begin{array}{ccc}
p_{1} & 0 & k \\
1 & p_{2} & 0 \\
0 & 1 & 0
\end{array}\right]}
\end{aligned}
$$

4. Transfer Function with 2 Poles 1 Zero

$$
\frac{Y(s)}{U(s)}=\frac{\left(s-z_{1}\right)}{\left(s-p_{1}\right)\left(s-p_{2}\right)} k
$$




$$
\left[\begin{array}{ll}
\mathbf{A} & \mathbf{B} \\
\mathbf{C} & \mathbf{D}
\end{array}\right]=\left[\begin{array}{ccc}
p_{1} & 0 & k \\
p_{2}-z_{1} & p_{2} & 0 \\
1 & 1 & 0
\end{array}\right]
$$

5. Transfer Function with 2 Poles 2 Zeros

$$
\begin{gathered}
\frac{Y(s)}{U(s)}=\frac{\left(s-z_{1}\right)\left(s-z_{2}\right)}{\left(s-p_{1}\right)\left(s-p_{2}\right)} k \\
{\left[\begin{array}{cc}
\mathbf{A} & \mathbf{B} \\
\mathbf{C} & \mathbf{D}
\end{array}\right]=\left[\begin{array}{ccc}
p_{1} & 0 & k \\
p_{2}-z_{1} & p_{2} & 0 \\
\sum_{i=1}^{2}\left(p_{i}-z_{i}\right) & p_{2}-z_{2} & k
\end{array}\right]}
\end{gathered}
$$

6. Transfer Function with 3 Poles 0 Zeros

$$
\begin{gathered}
\frac{Y(s)}{U(s)}=\frac{1}{\left(s-p_{1}\right)\left(s-p_{2}\right)\left(s-p_{3}\right)} k \\
{\left[\begin{array}{ll}
\mathbf{A} & \mathbf{B} \\
\mathbf{C} & \mathbf{D}
\end{array}\right]=\left[\begin{array}{cccc}
p_{1} & 0 & 0 & k \\
1 & p_{2} & 0 & 0 \\
0 & 1 & p_{3} & 0 \\
0 & 0 & 1 & 0
\end{array}\right]}
\end{gathered}
$$

7. Transfer Function with 3 Poles 1 Zeros

$$
\begin{gathered}
\frac{Y(s)}{U(s)}=\frac{\left(s-z_{1}\right)}{\left(s-p_{1}\right)\left(s-p_{2}\right)\left(s-p_{3}\right)} k \\
{\left[\begin{array}{ll}
\mathbf{A} & \mathbf{B} \\
\mathbf{C} & \mathbf{D}
\end{array}\right]=\left[\begin{array}{cccc}
p_{1} & 0 & 0 & k \\
p_{2}-z_{1} & p_{2} & 0 & 0 \\
1 & 1 & p_{3} & 0 \\
0 & 0 & 1 & 0
\end{array}\right]}
\end{gathered}
$$

8. Transfer Function with 3 Poles 2 Zeros

$$
\begin{gathered}
\frac{Y(s)}{U(s)}=\frac{\left(s-z_{1}\right)\left(s-z_{2}\right)}{\left(s-p_{1}\right)\left(s-p_{2}\right)\left(s-p_{3}\right)} k \\
{\left[\begin{array}{cc}
\mathbf{A} & \mathbf{B} \\
\mathbf{C} & \mathbf{D}
\end{array}\right]=\left[\begin{array}{cccc}
p_{1} & 0 & 0 & k \\
p_{2}-z_{1} & p_{2} & 0 & 0 \\
p_{3}-z_{2} & p_{3}-z_{2} & p_{3} & 0 \\
1 & 1 & 1 & 0
\end{array}\right]}
\end{gathered}
$$

9. Transfer Function with 3 Poles 3 Zeros

$$
\begin{gathered}
\frac{Y(s)}{U(s)}=\frac{\left(s-z_{1}\right)\left(s-z_{2}\right)\left(s-z_{3}\right)}{\left(s-p_{1}\right)\left(s-p_{2}\right)\left(s-p_{3}\right)} k \\
{\left[\begin{array}{cc}
\mathbf{A} & \mathbf{B} \\
\mathbf{C} & \mathbf{D}
\end{array}\right]=\left[\begin{array}{cccc}
p_{1} & 0 & 0 & k \\
p_{2}-z_{1} & p_{2} & 0 & 0 \\
p_{3}-z_{2} & p_{3}-z_{2} & p_{3} & 0 \\
\sum_{i=1}^{3}\left(p_{i}-z_{i}\right) & \sum_{i=2}^{3}\left(p_{i}-z_{i}\right) & p_{3}-z_{3} & k
\end{array}\right]}
\end{gathered}
$$


